\title{
Primeros resultados de la excavación del castillo medieval de Dos Hermanas (Montemayor, Córdoba)
}

First results of the excavation of the medieval castle of Dos Hermanas (Montemayor, Cordoba)

\author{
Javier López Rider a , Santiago Rodero Pérez ${ }^{b}$, José Manuel Reyes Alcalác \\ a Área Historia Medieval - Universidad de Córdoba, Córdoba, Spain, lopezrider@uco.es

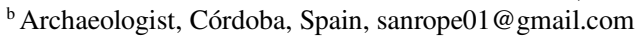 \\ ${ }^{\mathrm{c}}$ Architect, Córdoba, Spain, reyes.gestor@gmail.com
}

\begin{abstract}
In the south of the kingdom of Córdoba, there is the castle so-called Dos Hermanas, located in the municipality of the current town of Montemayor. It has been considered that the construction of the castle of this stately town was the result of the first moments of decline of the fortress of Dos Hermanas, located on the bank of the Carchena stream. Currently, a first excavation campaign has been carried out that brings us closer to the anthropic occupation of the site. At the same time, the archival research gives new information to the history of the site, exceeding the date of 1340, when Don Martín Alonso de Córdoba partially destroyed the Arab fortress of Dos Hermanas to build the castle of Montemayor. The first data extracted from the field work support the written sources, providing us with new data that allow us to make a more complete and novel interpretation. The survival of part of the facilities of the Dos Hermanas castle with an occupation from Roman times to the sixteenth century that shows the total nondepopulation of the place in the fourteenth century, as previously thought. A high degree of conservation of the structures found inside the wall enclosure appears a southern bay with stables with nine mangers. To the west, there is a vain and an angled staircase that allowed access from the parade ground until the round pass over the main door, which is also preserved. The objective of this proposal will be to present these first results of the archaeological intervention centered on the southern wall of the castle. These research works are accompanied by a consolidation project of the main structures, all financed by the Provincial Delegation of Cordoba and Montemayor Town Hall, whose continuity is developed in 2019 and 2020.
\end{abstract}

Keywords: Middle Ages, Kingdom of Cordoba, castellology, medieval archeology.

\section{Introducción}

Actualmente existe un castillo denominado Dos Hermanas, ubicado en el término municipal del pueblo de Montemayor. Hasta el momento se ha interpretado que, a consecuencia de la decadencia de la fortaleza de Dos Hermanas, situada en la orilla del arroyo Carchena, se aprovecharon sus materiales para edificar un nuevo castillo en la villa de Montemayor a mediados del siglo XIV. Recientemente se ha llevado a cabo una primera campaña de excavación que, junto a la investigación archivística desarrollada, dota de nueva información histórica del recinto, permitiendo descartar dicha teoría sobre su destrucción y despoblamiento en 1340. Según se ha po- 
dido dilucidar, don Martín Alonso de Córdoba no destruyó el castillo de Dos Hermanas para levantar su castillo señorial en Montemayor. Los resultados de esta campaña de excavación nos muestran la pervivencia de parte de las instalaciones del castillo de Dos Hermanas con una ocupación ininterrumpida desde época prerromana hasta el siglo XVI (Fig. 2). Se pone de manifiesto que la hipótesis sobre una total despoblación del lugar en el siglo XIV, es infundada. De hecho, se constata un grado de conservación elevado de las estructuras halladas en el interior del recinto murario (Fig. 2), que igualmente la investigación consideraba arrasado y amortizado hasta sus cimentaciones. Actualmente, salvo las torres y la puerta, el resto se mantiene en un estado más que aceptable (Fig. 1). A tenor de los resultados de estas primeras intervenciones podemos concretar la siguiente secuencia de ocupación plurisecular.

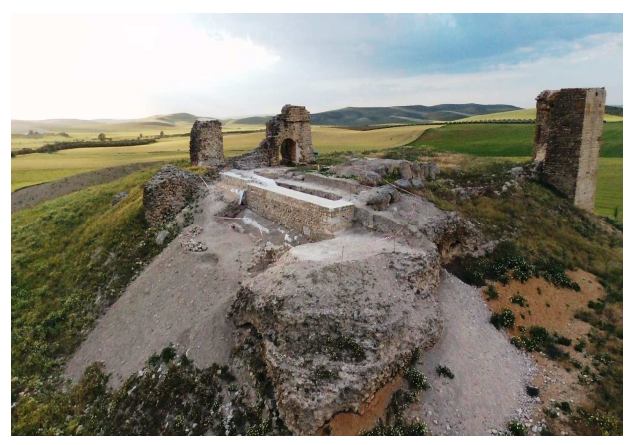

Fig. 1. Vista general del castillo de Dos Hermanas hoy día (Autores, 2019).

\section{Evolución Histórica}

\subsection{Fase prerromana}

El primer momento de ocupación antrópica viene definido por la presencia dispersa de fragmentos cerámicos y pétreos de época calcolítica. Este periodo se desarrolló en nuestro ámbito de estudio entre el Neolítico y la Edad del Bronce, en torno al II milenio a.C. El investigador Morena, ya puso de manifiesto la presencia de este tipo de vestigios en el ruedo del castillo de Dos Hermanas, catalogándolo según el análisis del material superficial, como un asentamiento de primer orden para el caso de Montemayor y de buena parte de la Campiña cordobesa (Morena, 1996, p. 105). Un emplazamiento privilegiado por su cercanía a un curso de agua, arroyo de la Carchena, y la existencia de tierras fértiles en su entorno. Concluía Morena en su estudio que la ausencia, al menos en superficie, de las típicas cazuelas carenadas de inicios del Cobre, entre otros factores, permiten establecer el primer asentamiento humano hacia el 2500 a.C.

El periodo íbero viene representado por hallazgos de tipo cerámico, dispersos y poco abundantes. Se localizan en las laderas del recinto fortificado, sin que en la excavación hayan aparecido en los estratos de ocupación más antiguos bienes muebles que nos hablen de niveles de ocupación prerromana, ni en fosas de cimentación, ni por remoción de capas históricas por la acción de la fauna. Esta ausencia contrasta con lo que sabemos de intervenciones anteriores en que se dibujaron y fotografiaron "figuras de platos y ánforas, de la edad del Bronce, ibéricas, romanas, califales y de los siglos XIV al XVII" (Moyano, 1986 , p. 65). Aun cuando tradicionalmente diferentes autores identificaban a Dos Hermanas con la antigua ciudad romana de Soricaria (Oliver, 1861, p. 73; Fernández-Guerra, 1866, p. 6), población que fue escenario de un cruento episodio bélico acaecido el 5 de marzo del 45 a.C. (Bell. Hisp. XXIV), ya ha sido sistemáticamente descartada por la historiografía actual. Se ha desestimado que Soricaria proceda del vocablo latino Soroa que tiene vinculación con los dos términos Dos y Hermanas. Incluso que Sor y Hermana son sinónimos en castellano y en latín (López de Cárdenas, 1773, p. 71; Morena López, 1998, p. 33; Moyano Llamas, 2000, pp. 86-87). Afirmación que ha podido ser corroborada por los recientes hallazgos localizados en la intervención arqueológica.

Del análisis de la ortofotogrametría del cerro del castillo, se deduce un importante conjunto de estructuras circulares y rectangulares, realizadas en piedra que la actual vegetación apenas permite vislumbrar en superficie. Estas formas dispersas por el cerro, en especial en las zonas noroeste y sureste, no presentan analogías respecto a las estructuras defensivas del recinto fortificado de Dos Hermanas, evocando más la presencia de un posible asentamiento tipo hábitat de planta circu- 
lar con zócalo de piedra, sin compartimentación interior, con varios metros de diámetro y con una dispersión aleatoria. Estas estructuras, situadas en lo alto del cerro y construidas con piedras, en principio no parecen que dispongan de un foso tipo rondels.

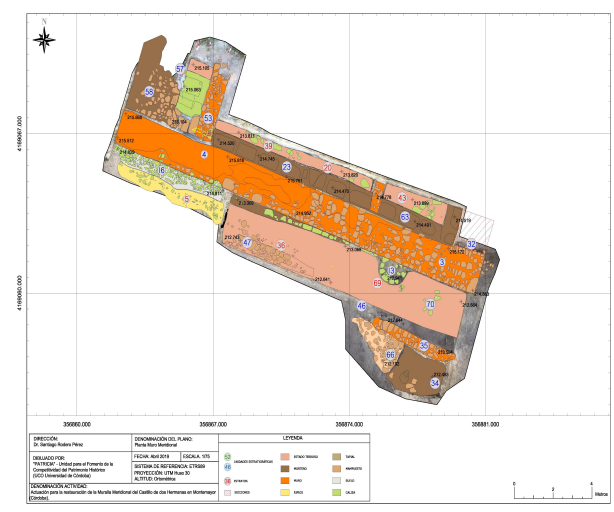

Fig. 2. Planta general de las estructuras documentadas en la campaña 2019 del castillo de Dos Hermanas (Autores, 2019).

Tampoco es posible, hoy por hoy, concretar si las estructuras cuadradas que se aprecian en superficie sean recintos fortificados de cantería de aquellas fases más evolucionadas.

\subsection{Fase romana}

Respecto de la ocupación romana documentada en la intervención, se caracteriza por la existencia, en la falda sur del cerro del castillo, de un depósito de opus caementicium ubicado en la margen derecha del arroyo de la Carchena, cuya función era la de almacenar agua para los usos de un hábitat rural próximo. Habría que ponerlo en relación con la localización de los vestigios romanos documentados en el cerro a cota 214 m.s.n.m. El asentamiento romano localizado en la excavación dominaba terrenos fértiles a su alrededor, con abundante agua (arroyo Carchena y fuente de Dos Hermanas), la presencia de una antigua vía de comunicación (actual camino de Duernas) que comunicaba la villa de Montilla (identificada como Munda) con Ategua, por donde dicho camino cruza el arroyo Carchena, convirtiéndose durante las épocas ibérica, roma- na y medieval en un punto estratégico de control del territorio.

Los vestigios más significativos de esta etapa son: un muro de opus quadratum arrasado, orientado este-oeste, una zona abierta a modo de calle pavimentada de gravas y guijarros, un pozo y la presencia de cerámica de almacenamiento. En la falda de poniente del cerro se localizan muros y frentes de cantera que podemos vincular igualmente a este momento histórico. Las huellas romanas localizadas nos hablan de la presencia, apenas apuntada, de una ocupación romana de carácter agropecuario. Probablemente se trate de un asentamiento rural, cuyas estructuras estarían en relación con la parte frumentaria de una villae, cuya pars rústica se encontraría en este punto del altozano. Apenas un fragmento de tesella y un ladrillo con forma de cola de milano, ambas piezas habitualmente relacionadas con espacios termales y habitados, pavimentados con opus tesellatum. Esto nos permite verificar la existencia en las cercanías de una posible villa romana. Hasta el momento no contamos con más datos de esta etapa histórica.

\subsection{Fase tardoislámica}

En relación a la fundación del castillo de Dos Hermanas, tanto su origen como su evolución, se constata con la presencia de un primer ḥiṣn islámico. Cronológicamente puede ser situado dentro del periodo de consolidación territorial y fronterizo llevado a cabo desde la fitna, en época almorávide y posteriormente por los almohades entre finales del siglo XI y principios del siglo XIII. La inexistencia de los tradicionales verdes manganesos de época califal y, la constatación en esta primera aproximación de técnicas constructivas en tapial, semejantes a las desarrolladas por los almorávides y los Unitarios en la provincia cordobesa, El Vacar o Palma del Río, entre otros (Córdoba, 2004, pp. 123-130), nos hace conjeturar que el origen del castillo se relaciona con esos momentos convulsos de al-Andalus. A falta de un estudio pormenorizado del material cerámico encontramos, al exterior del muro sur, formas y decoraciones almorávides de trazos digitales en manganeso junto a fragmentos de azul turquesa o cerámica más tardía como jofainas y 
ataifores melados con manganeso. Por lo que, a la edilicia de este primer momento, se ha documentado un lienzo, en la vertiente sur del cerro, de casi $15 \mathrm{~m}$ de longitud que se desarrolla en dos cuerpos. El sector inferior se conforma como un paramento longitudinal a modo de zócalo de piedra formado por mamposterías careadas y sillarejos escuadrados sobre el que se asientan unas cajas de tapial. Estas tapias se elevan verticalmente formando la cortina sur del castillo original. Delante de la muralla meridional y, a unos $2 \mathrm{~m}$ de distancia, se ha constatado la presencia de un antemuro de tapia que se cimentó sobre una losa de mampostería. Este muro delantero se distribuye longitudinalmente adaptándose al terreno por la ladera sur como una primera línea defensiva. La fundación de esta falsabraga de tapia supuso la ruptura del suelo de gravas preislámico. Por su parte, este primer lienzo amurallado del sur del castillo, cimienta sobre las estructuras romanas documentadas. Hacia el interior de este muro se constata que el nivel de suelo está por encima de la cota de arranque de la cimentación llevándonos a definir un escalonamiento entre el suelo original intramuros y la liza. Esta liza o camino perimetral localizado entre el antemuro y el muro sur transcurriría entre la puerta oeste y la torre principal en el sureste.

Es probable que, la construcción y fundación de este recinto fortificado islámico de Dos Hermanas, pudiera incluirse en los dos grandes momentos de época tardoislámica. O bien con la llegada de las huestes almorávides y la consolidación de su territorio a principios del siglo XII, o en un proceso constructivo auspiciado por la eclosión constructiva almohade que se desarrolla por toda la Campiña occidental (Márquez Bueno, 2018, pp. 11 y ss.). Respecto a la primera posibilidad podríamos contextualizar su construcción dentro de los principales conflictos heredados por los almorávides en Qurțuba a consecuencia de las relaciones de los Abadíes respecto de los Ziríes de Granada, Málaga o los Banu sumadih de Almería. La máxima extensión del imperio almorávide alcanzada a principios del siglo XII llegando hasta Zaragoza, vendría aparejada de la instalación de fortines que controlasen la vías, arroyos y pasos principales como los existentes en las cercanías del cerro de Dos Herma- nas. En lo referente al segundo, realizado por los almohades a partir de la mitad del siglo XII, la importancia de Córdoba para el nuevo regidor lo ponen de manifiesto las fuentes cuando reconvierte temporalmente a Qurțuba (Pabón Fernández, 2016, p. 195; Viguera Molis, 1997, p. 88) como capital de al-Andalus y en primera línea de defensa hacia el norte (León Muñoz, et al., 2008, pp. 261 y ss.). La ubicación del castillo de Dos Hermanas se debe a los conflictos contra los reinos cristianos y con otras líneas de frontera. Las principales vías de conflicto entre cristianos y almohades formaban una pinza desde Calatrava la Vieja, Andújar, Valle del Guadalquivir y desde Badajoz, Montemolín y Palma del Río (Pabón, 2016, p. 196). En este contexto territorial el castillo objeto de estudio se halla en la margen izquierda del Guadalquivir y su valle, en plena Campiña y de espaldas a estas zonas rojas, por lo que su construcción debió de responder a la defensa del territorio de razias o conflictos por el sureste. En este momento, los peligros por oriente solo podían proceder de problemas o enfrentamientos con las Coras de Elvira o de Bayyana y a este peligro respondería la construcción del recinto.

La fortaleza de Dos Hermanas cuenta con una posible entrada en recodo por el suroeste con una torre albarrana en este sector, uso generalizado del tapial como material constructivo, basamento de piedra y un posible antemuro. Según lo avanzado en la excavación, parece que carece de corachas, torres poligonales, encadenados de piedra en las esquinas y falta de monumentalidad del acceso. En cuanto al tratamiento epidérmico de los tapiales no hemos documentado en esta primera campaña la decoración de falsos despieces de sillerías o encintados calizos y acabados superficiales (Márquez Bueno, Gurriarán Daza, 2008, p. 117). El análisis de los paramentos como el de los materiales cerámicos no aporta en esta primera campaña más información de esta fase tardoislámica.

Si se comparan los datos obtenidos en paralelo con otras construcciones similares, nos topamos con la presencia de ciertas semejanzas constructivas de la obra de cantería y tapia de fortificaciones como las de la Alcazaba de Palma del Río (Pabón, 2016, p. 241) o en Reina (Márquez, 
2018, p. 13) que presentan refuerzos de piedra en las esquinas o alternan zarpas o basamentos de sillares o ladrillos sobre las que se eleva el tapial. A falta de datos cronotipológicos más claros, la datación almohade de estos recintos se puede encontrar en diversos trabajos (ej., Pavón, 1999, vol. 3, p. 125), que motivan que no descartemos su filiación almohade. El análisis de las estructuras de tapial esparcidas por el yacimiento, tanto las ubicadas en el lugar como las que se encuentran dentro de las torres noreste y el lienzo este, nos muestran cómo en época bajomedieval, se produce una gran reforma de los muros quedando integrados en torres y al interior de un recinto de sillería mucho más amplio. Quizás este hịṣn fue uno de aquellos que constituiría las veintisiete fortalezas entregadas por los musulmanes a Fernando III el Santo al reconquistar las tierras cordobesas.

\subsection{Fase bajomedieval}

La ocupación de la original fortificación islámica por las huestes de Fernán Núñez de Témez (Fernández de Bethencourt, 1905) supuso el segundo momento edilicio documentado en el castillo de Dos Hermanas. Se produce en esta etapa una gran actividad constructiva, cuyo resultado más evidente, es la amortización de las torres originales de tapia mediante la construcción de retobos de sillarejos que las forran y engrandecen. En época bajomedieval el castillo alcanza unas dimensiones considerables, integrando en sus muros y en su interior las estructuras de épocas preexistentes. Se supera la cerca original y se conforma un recinto fortificado con tres líneas defensivas por su vertiente sur y oeste y una torre albarrana que flanquea un posible acceso en recodo. Este perímetro fortificado "en altura" dominaba las tierras de cultivo y las cercanas aldeas de Mingohijo (Domingo Hijo), La Parrilla del villar de San Pedro o Dos Hermanas (López Rider, 2017, tomo II, p. 644). El castillo estudiado está organizado en torno a un patio de armas, espacio central rodeado por altas murallas con cortinas interiores. Previsiblemente una de las cercas exteriores con que contaba el recinto fortificado, al exterior de la puerta, por su lado de poniente, definía una albacara o espacio de acogida de los aldeanos ante peligros exógenos. La puerta documentada nos lega como dato más significativo su orientación hacia occidente y la constatación de una posible luz de $3 \mathrm{~m}$ desde el trasdós del arco hasta el suelo original. Esta entrada estaba defendida, a su izquierda, por una serie de cortinas alineadas con ella que conseguían ceñirla.

En las curvas de nivel de menor altimetría del cerro se construye una primera línea defensiva hacia el sur, a modo de cerca, a cota de 210 m.s.n.m., seguida por un antemuro de varios metros de altura y unos $0,60 \mathrm{~m}$ de grosor que se eleva como defensa frontal de sus respectivos lienzos. Por último, la muralla interior presenta varios metros de altura, $1,05 \mathrm{~m}$ de grosor y torres cada $15 \mathrm{~m}$, que se levantan entre forros de mampostería y tapial. En la esquina sureste sobre las estructuras amortizadas de la torre de tapial del primigenio recinto islámico, se construye y cimienta la Torre del Homenaje de $6 \mathrm{~m}$ de planta que se encuentra arrasada. Enlazaba con la torre oeste de la puerta mediante una falsabraga de mampostería apoyada sobre tapial de época islámica. Al interior del castillo se encontrarían diversas dependencias adosadas a la muralla (habitaciones de los que trabajaban y vivían en él, talleres de diversos oficios, almacenes, un aljibe o las cuadras). Las caballerizas, en un gran estado de conservación, se localizaron al interior del muro sur con pesebres de obra y muros separadores de estancias. La pared interna se enjalbega y se instalan abrevaderos de piedra revestidos con morteros de cal. Respecto a las cuadras, en época andalusí, Ibn al-Awwam el Awan (Banqueri, 1802, pp. 525-527) consigna en su Libro de Agricultura, tomo segundo, capítulo 32 y artículo XVI, algunas consideraciones sobre la construcción y acondicionamiento de aquellas. Unas recomendaciones que se han constatado en las caballerizas excavadas en Dos Hermanas (Fig. 4). Por último, de esta fase destaca la aparición de una escalera (Fig. 3) en ángulo recto que, flanqueando el acceso principal por su lado sur, giraba en ascenso hasta disponerse sobre el paso de ronda del lienzo murario oeste.

El tercer momento constructivo se produce ya en pleno periodo bajomedieval y viene definido por la compartimentación de espacios al interior del castillo y la reparación de las caballerizas. Esta 
obra se realiza con morteros blancos y rosados, similares a los localizados en el castillo de Aguilar de la Frontera o el de Iznájar (Rodríguez Aguilera, Bordes García, 2009) para los siglos XIV y XV. La presencia de numerosa cerámica de Manises, formas de cuencos y escudillas de loza y vedríos verdes con asas decoradas, nos hablan de una ocupación plurisecular ininterrumpida desde el siglo XII hasta el XVI. Este dato viene a cuestionar la teoría tan extendida del abandono, en el siglo XIV, del castillo de Dos Hermanas para la construcción del correspondiente a la villa señorial de Montemayor. Casas-Deza, afirmaba que don Alonso Fernández de Córdoba, dejó a su hijo don Martín Alonso de Córdoba el castillo de Dos Hermanas, "situado a unas 6 leguas al mediodía de Córdoba y como esta fortaleza estuviese en paraje poco proporcionado para defenderla, la demolió y edificó otra en monte cercano de mayor elevación en 1340 , en cuyo año el rey Don Alonso XI, le concedió privilegio para que fundase allí población como heredad suya que era, por lo cual la nueva villa se denominó Montemayor" (Casas-Deza, 1986, tomo II; Abad de Rute, 1965-1967, pp. 485-497).

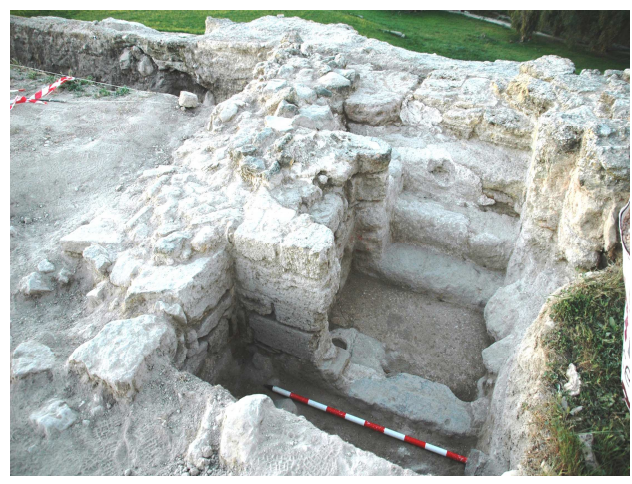

Fig. 3. Escalera que da acceso al paso de ronda del muro oeste (Autores, 2019).

A este respecto debemos de apuntar que, aunque la solicitud de don Martín Alonso de Córdoba se realiza en 1340 entendemos que dicho requerimiento no conllevó el inicio inmediato de la construcción del castillo de Montemayor y mucho menos la demolición del de Dos Hermanas.
La supuesta hipótesis de la construcción del castillo de Montemayor en detrimento del recinto de Dos Hermanas, es más que cuestionable, pues no solo no se ha constatado la presencia de un desmonte sistemático (horizontes de saqueo, acopio de materiales, restos de morteros o sillares) sino que sabemos que, en este momento, se reforman o reparan las caballerizas y otras estancias del castillo, apareciendo ajuar de mesa, cocina y almacenamiento de cerámica del siglo $\mathrm{XV}$, como hemos mencionado. Incluso las fuentes existentes en el Archivo Catedralicio nos hablan del pago de prestameras y tercias por parte de la parroquia del núcleo poblacional de Dos Hermanas hasta 1498 como mínimo, lo que supone la presencia estable de un hábitat vinculado al castillo (López, 2017, tomo I, p. 310 y ss.).

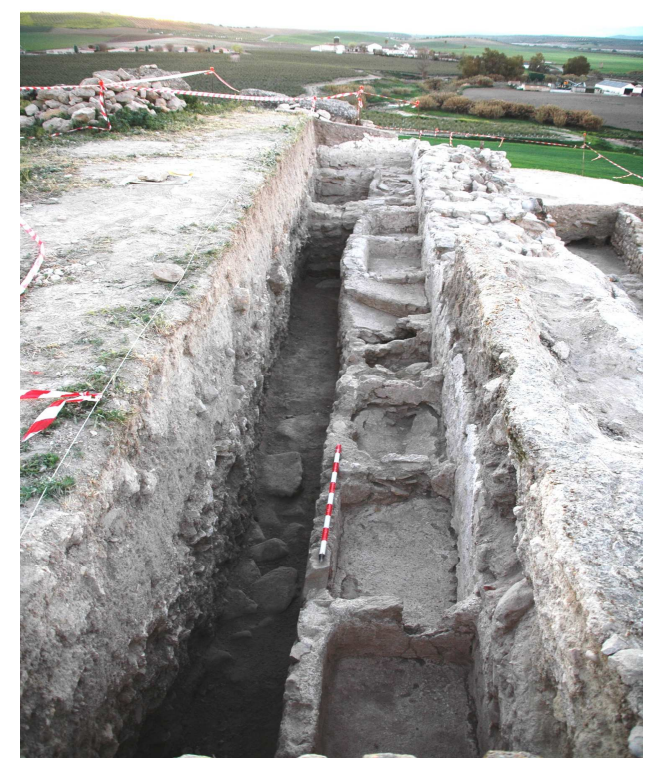

Fig. 4. Pesebres de las cuadras excavadas (Autores, 2019).

\subsection{Fase Moderna - Contemporánea}

Asistimos en este momento a un olvido parcial del conjunto, aun cuando la finca conserva el nombre de "Cortijo de Dos Hermanas". Consecuentemente este espacio se convierte en predio agrario de éxito para la población de Montemayor. Los muros en alzado todavía visibles y utilizados como canterías cerraban una zona para 
pasto de ganadería caprina hasta fechas recientes cuando el Ayuntamiento de Montemayor adquiere la propiedad para su protección y restauración.

\section{Conclusiones}

Se han plasmado los primeros resultados, pues queda afortunadamente mucho por excavar y conocer. Uno de los resultados que más ilusión genera es la constatación de niveles de suelo en torno a los $-1,80 \mathrm{~m}$ respecto de la cota actual de suelo, lo que se traduciría en la presencia de estancias con al menos $1,50 \mathrm{~m}$ de alzados conservados. Esto es realmente positivo pues significa que el arrasamiento del castillo no es así en todos los sectores y que su futura valorización podría contar con espacios intramuros originales: vanos, tabiques, abrevaderos, suelos, etc. Igualmente queda por definir la planta exacta del recinto fortificado, de las líneas defensivas perimetrales adelantadas en las laderas sur y oeste, de las torres de la posible entrada en recodo, de la disposición de la torre albarrana, entre otros elementos.

La intervención en campo nos aporta la ocupación plurisecular del yacimiento que abarca diferentes momentos a lo largo de los siglos de su existencia: fase romana, islámica, medieval cristiana, moderna y contemporánea.

Los principales vestigios localizados, según un criterio cronológico fueron: aparición de restos de época prerromana en la excavación, amortizados y sirviendo de cimentación al muro sur del castillo. Se constata por fotogrametría la presencia de estructuras circulares y regulares de piedra por todo el sector sur y oeste del cerro previsiblemente de época protohistórica.

Respecto a la fundación del castillo es de indudable factura islámica, entre los siglos XII y XIII. De este momento se conserva el zócalo de piedra y parte del alzado de tapial de la planta y el alzado del muro sur del castillo. También se han constatado la existencia de vestigios de un antemuro de tapial al sur del castillo. En un momento bajomedieval cristiano, se produce el otro gran momento constructivo del castillo, ampliándose las defensas, restaurando y exterior un antemuro, que rompe el anterior de tapial y la posible liza. Al interior del patio de armas se documentó una crujía usada como caballerizas. englobando las islámicas en un recinto fortificado de mayor entidad, realizado en cantería. De este periodo se ha documentado al Estas se dividieron originalmente en dos estancias y presenta hasta nueve pesebres, en buen estado, conservándose el suelo de lajas y tierra original. Se constató la presencia de unas escaleras que permitían acceder desde el patio de armas hasta el paso de ronda en la esquina suroeste de la cortina sur del castillo.

Por lo que respecta a la amortización del castillo en época posterior se ha documentado cerámica de los siglos XV y XVI, en estratos de colmatación y abandono de las caballerizas que nos dan una fecha del uso de las mismas. Diferentes alineaciones de ripios y mampuestos por el interior del castillo nos hablan de reutilización tardía de las instalaciones del mismo con usos posteriores de carácter rural.

Desde el siglo XVII se confirma el abandono, saqueo y desmonte de parte de las estructuras del castillo y la progresiva colmatación por tierras posteriormente cubiertas por manto boscoso. Los datos que se están recopilando de la documentación bajomedieval y el siglo XVI, respaldan en su mayor parte, los vestigios localizados hasta el presente. Gracias a la apuesta del Ayuntamiento de Montemayor, se está conociendo un castillo medieval inédito de la Campiña cordobesa, cuya puesta en valor está aportando interesantes resultados.

\section{Bibliography}

Abad de Rute. (1965-1967). "Historia de la Casa de Córdoba", Boletín de la Real Academia de Córdoba, 87, pp. 485497.

Banqueri, J.A. (1802). Kitab al-falahah. Libro de agricultura. Su Abu Zacaria Jihia aben Mohamed ben Ahmed Ebn el Awam, Sevillano, Banqueri, J.A. trad., Imprenta Real, Madrid, vol. 1. 
Casas-Deza, L.Mª de. (1986). Corografía histórico-estadística de la provincia y obispado de Córdoba, 2 tomos, Córdoba.

Córdoba De la Llave, R. (2004). "Fortificaciones almohades de la provincia de Córdoba", in Valor, M.; Villar, J.L.; Ramírez, J., Los Almohades. Su patrimonio arquitectónico y arqueológico en el sur de al-Andalus, Consejería de Relaciones Internacionales D. L., Sevilla, pp. 123-130.

Fernández de Bethencourt, F. (1905). Historia genealógica y heráldica de la monarquía española, Madrid, vol. 6.

Fernández-Guerra, A. (1866). Munda pompeyana, Imprenta y estereotipia de M. Rivadeneyra, Madrid.

León Muñoz, A.; León Pastor, E.; Murillo Redondo, J.F. (2008). "El Guadalquivir y las fortificaciones urbanas de Córdoba", in Actas del IV Congreso Internacional sobre fortificaciones: "Las fortificaciones y el mar", Alcalá de Guadaíra (Sevilla), pp. 261-290.

López de Cárdenas, F.J. (1773). Memorias antiguas de algunas poblaciones de la Bética, de su topografía e inscripciones inéditas o erradas, Parte Segunda, Montoro.

López Rider, J. (2017). Santaella y el suroeste de la Campiña cordobesa en la Baja Edad Media, 2 tomos, Colección de tesis doctorales del Repositorio de Helvia de la Universidad de Córdoba, Córdoba.

Márquez Bueno, S.; Gurriarán Daza, P. (2008). "Recursos formales y constructivos en la arquitectura militar almohade de al-Andalus", Arqueología de la Arquitectura, 5, pp. 115-134.

Márquez Bueno, S. (2018). "La tecnología constructiva andalusí: obra encofrada y revestimientos en la arquitectura militar (ss. XI-XIII). El ejemplo de las torres”, Arqueología de la arquitectura, 15, pp. 1-33.

Morena López, J.A. (1996). "Las primeras culturas de productores en la campiña de Montemayor (Córdoba): el poblado calcolítico de Dos Hermanas", Boletín de la Real Academia de Córdoba de Ciencias, Bellas Letras y Nobles Artes, 130, pp. 97-124.

Morena López, J.A. (1998). "Reflexiones sobre el emplazamiento de Soricaria (Bellum Hispaniense, XXIV y XXVII). Una nueva propuesta: El Cerro de las Cuevas de Sequeira (Castro del Río-Nueva Carteya. Córdoba)", Antiquitas, 9, pp. 31-44.

Moyano Llamas, P. (1986). "Lugares arqueológicos de Montemayor", in Notas para la historia de Córdoba y su provincia, Asociación provincial cordobesa de cronistas oficiales, Córdoba, pp. 63-66.

Moyano Llamas, P. (2000). "Excavaciones en Dos Hermanas", Boletín de la Real Academia de Córdoba de Ciencias, Bellas Letras y Nobles, 139, Córdoba, pp. 85-94.

Oliver, J.M. (1861). Munda pompeyana, Imprenta de Manuel Galiano, Madrid.

Pabón Fernández, Ma.P. (2016). “Arquitectura defensiva almohade en la provincia de Córdoba. Los ejemplos de Hornachuelos y Palma del Río", Anhagramas, 2, pp. 188-270.

Pavón Maldonado, B. (1999). Tratado de arquitectura hispanomusulmana. Fortalezas, CSIC, Madrid, vol. 3.

Rodríguez Aguilera, A.; Bordes García, S. (2009). Memoria preliminar de la Actividad Arqueológica Puntual "Castillo de Iznajar”, Iznájar.

Viguera Molins, M.J. (1997). "Los Almohades", in Viguera Molins, M.J. coord., El retroceso territorial de Al-Andalus. Almorávides y Almohades. Siglos XII al XIII, serie Historia de España, Madrid, tom. VIII-XII, pp. 73-111. 